\title{
FLOW INJECTION ANALYSIS IN ON-LINE PROCESS CONTROL
}

\section{W. E. VAN DER LINDEN}

Laboratory for Chemical Analysis, Department of Chemical Technology, Twente University of Technology, P.O. Box 217, 7500 AE Enschede (The Netherlands)

(Received 3rd July 1985)

\section{SUMMARY}

Flow injection systems are serious candidates for a new generation of chemical on-line analyzers because there is a growing interest in instruments that combine versatility with the possibility of attaining high sampling frequencies. For real on-line applications the instrument and its component parts have to meet the highest standards with respect to reliability and maintenance. These aspects are considered in some detail, and some industrial applications are briefly discussed.

Most process plants in operation today are still primarily controlled by monitoring operational variables such as temperature, pressure, flow and liquid level. These variables can be measured reliably and the equipment needed is relatively easy to install, calibrate and maintain. The failure rate and down-time of these devices is low, but, in general, this does not apply yet to the present generation of process analyzers, although a gradual improvement in the situation can be observed. The higher failure rate and the higher demands on maintenance of on-line analyzers is, of course, mainly due to the greater complexity of the equipment. Moreover, unlike temperature-measuring devices and pressure gauges, most of the sensing devices for chemical components cannot be installed directly in the process stream. This means that sampling and handling of sample streams form an integral part of the analyzing systems with all the complications inherent to it.

In spite of the difficulties encountered, there is a growing interest in process analyzers. The main reason is that by means of the analysis of the chemical coinposition of process streams the process operation can be "finetuned" to optimal levels not otherwise possible [1]. Optimization is of great importance for economic reasons, allowing better use of raw materials and energy as well as corrosion prevention; for environmental reasons, giving a check on the production of undesirable, toxic or otherwise hazardous byproducts and continuous monitoring of waste streams required by stringent statutory regulations; and for quality reasons, the higher quality demanded for products leading to a narrowing of the out-of-specification limits.

Although these remarks apply to continuous bulk processes, on-line analysis may be of even greater importance for processes that cannot be considered 
as pure formulation processes in which components are simply mixed together to arrive at the final product. So, especially in most of the biotechnical production processes, chemical analyzers are essential.

Because the number of different component analyzers available on the market is quite limited and because it is not to be expected that this situation will improve dramatically in the near future, it is very attractive to focus on flexible modular systems that can easily be adapted to achieve the desired type of analysis. It is within this framework that such versatile methods as air-segmented continuous flow analysis (c.f.a.) and flow injection analysis (f.i.a.) come into the picture, but the use of completely automated titrators has also to be taken into consideration. Analyzers based on airsegmented c.f.a. are commercially available (e.g., Technicon Monitor 650). The very long experience with similar equipment, in particular in clinical laboratories, has led to the development of dependable parts with which a great variety of set-ups can be constructed. Although it is true that f.i.a. has shown explosive growth over the last six years, and most of the individual component parts needed to build a flow injection system have been well tested over longer periods of time, hardly any information on the application of these systems in process analysis can be found in the literature. No multipurpose process analyzer based on this principle is available commercially. The object of this paper is to present a concise discussion of the advantages and disadvantages of f.i.a. for on-line process control.

\section{ANALYZER REQUIREMENTS}

In some respects the requirements for a process analyzer are different from those of the corresponding laboratory instrument. Some of these aspects will be considered in more detail.

\section{Sampling frequency and mechanical aspects}

The quality of information gathered with an analyzer with respect to the possibilities for process control can be described by introduction of the concept of "measurability" $[2,3]$. The mathematical expression for measurability $(m)$ comprises the time lag between sampling and result (delay time, $\left.t_{\mathrm{d}}\right)$, the time between consecutive samples $\left(t_{\mathrm{s}}=1 / f\right.$, in which $f$ is the sampling frequency), the time constant of both the process $\left(t_{\mathrm{p}}\right)$ and the measuring device $\left(t_{\mathrm{a}}\right)$ as well as the standard deviations for the analyzer proper $\left(\sigma_{\mathrm{a}}\right)$ and the relevant process variable $\left(\sigma_{\mathrm{p}}\right)$ :

$m \approx \exp \left[-\left(t_{\mathrm{d}}+1 / 2 t_{\mathrm{s}}\right) / t_{\mathrm{p}}\right]\left[1-\left(\sigma_{\mathrm{a}} / \sigma_{\mathrm{p}}\right)\left(t_{\mathrm{a}} / t_{\mathrm{p}}\right)^{1 / 2}\right]$

One of the important features of f.i.a. is its high sampling frequency. Therefore it has an advantage over, for instance, automated process titrators, if the process is subject to relatively rapid changes. A titrator might be preferable, however, for cases where process dynamics suggest that a somewhat lower sampling frequency $\left(f<\right.$ ca. 6 samples $\left.\mathrm{h}^{-1}\right)$ can be accepted but a higher precision of analysis is desirable. 
To benefit fully from the high sampling frequency, the components of the set-up have to fulfil demanding mechanical requirements. It has to be realized that 24-h sampling at 1 sample per 3 min means 175000 samples per annum. This emphasizes the general requirement that on-line analyzers should have as few moving parts as possible. In this respect, c.f.a. as well as f.i.a. compare favorably with titrators. In f.i.a., the injection valve is certainly the weakest part. A flow-injection analyzer for on-line process control should be constructed in such a way that maintenance and replacement of valves can easily be accomplished.

At present, reagent consumption in normal f.i.a. is generally of the order of $1 \mathrm{ml} \mathrm{min}$. However, on the basis of 24-h operation, this means a consumption of $500 \mathrm{l}$ per annum, which can represent a considerable expenditure. Particularly when expensive reagents have to be used, it is worthwhile considering the possibility of reversed f.i.a. [4-6], for which the reagent is injected into a continuous sample stream. The application of merging zones can be an alternative way to decrease reagent consumption but it has the disadvantage that either the injection valve must be more complex or that two valves have to be used simultaneously, both of which are mechanically less attractive options.

\section{Adjustment of temperature of sample and standards}

In order to provide accurate quantitative measurements, process-stream data analyzers have to be calibrated regularly. In the laboratory environment and particularly with batch samples, such calibration procedures seldom cause difficulties because standards and samples will have approximately the same temperature. Process streams, in contrast, can have temperatures that deviate significantly from that of the analyzer environment in which the calibration solutions are stored, and so will the process samples if no adequate precautions are taken.

The influence of variation in temperature on f.i.a. can be of two kinds. First, it affects the dispersion process by changing the diffusion coefficient value and by introducing temperature gradients. Secondly, it affects the rate of chemical reactions. This latter effect has only to be taken into consideration for those cases in which the height of the transient signal is largely determined by reaction kinetics. Apart from a paper by Fernandez et al. [7], little or no attention has been paid in the literature to the influence of temperature on dispersion in f.i.a. systems; it has always been assumed implicitly that all experiments are done at the same uniform temperature.

For a prediction of the influence of fluctuations of temperature of the whole system, a mathematical expression is needed for peak profile or peak height as a function of parameters for which the temperature dependence is well established. No such universally applicable expression is available, but if the axial dispersion model is adopted as a suitable approximation the following expression is valid:

$C_{\max }=\left(M / \pi R^{2}\right)\left(\langle v\rangle / 4 \pi L D_{L}\right)^{1 / 2}$ 
in which $M$ is the mass of material injected, $R$ is the tube radius, $\langle v\rangle$ is the mean linear flow velocity, $L$ is the tube length and $D_{L}$ is the so-called axial dispersion coefficient. At low Reynolds numbers, and for sufficiently long residence times to justify the assumption of Taylor flow conditions, $D_{L}$ can be expressed as a function of the molecular diffusion coefficient, $\mathbb{D}$, by

$D_{L}=R^{2}\langle u\rangle^{2} / 48 \mathbb{D}$

Substitution in Eqn. 2 leads to

$C_{\max }=\left(2 M / \pi^{3 / 2} R^{3}\right)(3 \mathbb{D} / L\langle v\rangle)^{1 / 2}$

Hence, $\mathrm{d} C_{\max } / \mathrm{d} T \approx \mathbb{D}^{-1 / 2} \mathrm{dD} / \mathrm{d} T$. Substitution of the Stokes-Einstein equation for the diffusion coefficient, $\mathbb{D}=k T / 6 \pi \eta r_{\mathrm{o}}$, where $\eta$ is the dynamic viscosity and $r_{\mathrm{o}}$ is the solute radius, yields the sought-for temperature dependence. However, it has to be realized that this does not present an unambiguous expression because viscosity also strongly depends on temperature as well as on the chemical composition of the fluid streams under study [8].

Difficult as it is to make some generally valid remarks on the influence of uniform temperature fluctuation of the whole assembly, even larger problems can be expected in making predictions when only the temperature of the sample plug is different to that of the rest of the equipment.

To conclude, it seems justified to state that in f.i.a. it is necessary to aim at the best possible uniformity of temperature between samples and standards. In c.f.a., where a steady-state situation exists at the moment of data collection, or in automatic process titrators, where equilibrium conditions prevail, fluctuations in temperature will have less influence. Moreover, in both latter methods the residence times are much longer, allowing for better heat exchange with the environment.

\section{TECHNIQUES BASED ON DISCRETE SAMPLES VS. CONTINUOUS ON-LINE} MEASURING DEVICES

In his discussion of the applications of f.i.a. in process analysis, Ranger [9] characterizes f.i.a. as a new approach for near real-time process monitoring. The expression near real-time might suggest that real-time measurement is an object to be pursued in process analysis. This is not generally true because it depends strongly on the time constant of the process, as discussed above. The faster the fluctuations in the process stream, the faster the analytical results have to be available. Nevertheless, it makes sense to raise this question in particular in relation to f.i.a. because, in principle, the same set-up can be used for real continuous measurements by leaving out the sample valve and introducing the sample stream continuously, giving an unsegmented continuous-flow system.

At first sight this might look an attractive proposal because it appears that the measurability will increase by decreasing $t_{\mathrm{s}}$ in Eqn. 1 to virtually zero. In fact, this is not true. By not using the transient character obtained with 
discrete sample plugs, data have to be collected under steady-state conditions, i.e., at a later stage. Because the delay time, $t_{\mathrm{d}}$, comprises both the time needed for transportation of the analyte from the process stream to the analyzer and the time spent inside the analyzer, data collection at a later stage means a corresponding increase in $t_{\mathrm{d}}$. Thus the effect of decrease in $t_{\mathrm{s}}$ is counterbalanced by the increase in $t_{\mathrm{d}}$ and there is no profit with respect to time to be gained by introducing the sample stream continuously instead of intermittently.

One important feature is eliminated by omitting the injection valve and that is the constant check on baseline drift (Fig. 1). By a proper selection of the injection frequency, a nearly complete return to the baseline can be achieved between two successive injections. In this way, it is easy to correct for slow fluctuations of the background. Moreover, with the elimination of the injection valve an important diagnostic tool will be lost. The profile of the transient signal obtained with plug injection can provide a trained operator with valuable information about the correct functioning of any flowinjection system.

\section{SAMPLING, SAMPLE TRANSPORT AND SAMPLE CONDITIONING}

Although f.i.a. is very well suited, in principle, for "continuous" on-line monitoring of liquid process streams, very few examples can be found in the literature. Most of them apply to the field of water quality and pollution monitoring; hardly any example deals with real process analysis. This is at least partly due to problems associated with the design and construction of adequate sampling and sample conditioning systems. In an excellent textbook on sampling systems for process analyzers, Cornish et al. [10] have presented a comprehensive survey of almost all the aspects related to sampling. In their introduction, they state that "...the complexities associated with sampling have often resulted in inadequate designs or have held back the use of on-line analysis, even though this may otherwise have been preferred to manual sampling and laboratory analysis procedures."

Some important requirements that the sampling system has to meet can
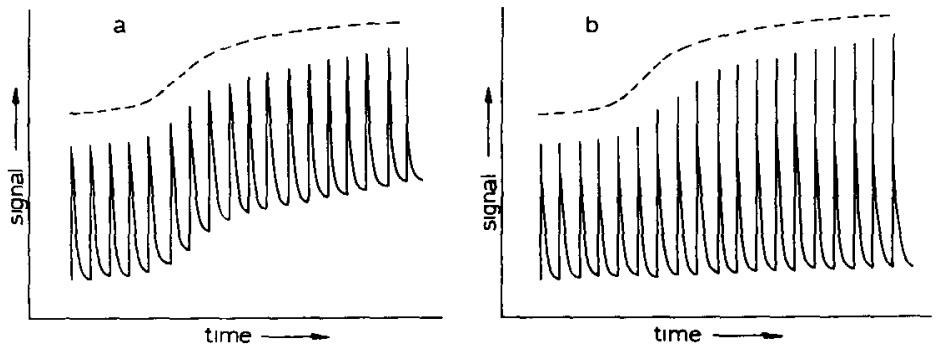

Fig. 1. Analyzer response vs. time (-) flow-injection device, (-...-) continuous monitoring device. (a) No concentration change; drift of baseline. (b) Concentration variation; constant baseline. 
be summarized as follows: removal of representative samples from the process stream and maintenance of the representativeness throughout the whole transport line; regulation of pressure and temperature of the sample provided to the analyzer; prevention of any vaporization, loss or contamination of the sample; quantitative transportation of the sample to the analyzer within a specified time period; provision of a means of introducing, when necessary, a calibration sample or standard; in a multi-stream application, provision of the ability to switch between sample streams without cross-contamination; provision of a means of returning part or all the sample to the process or to a waste container; removal of dirt and any other extraneous material from the sample; and provision of a quantitative means of sample dilution when necessary. Of course, not all these aspects are of interest for every analysis and for every case a thorough evaluation is necessary to assess which aspects deserve special attention, but the optimum design for a case at hand should be as simple as possible consistent with the required functions.

If the analyzer cannot be installed close to the process stream, a longer transport line with a correspondingly longer delay time may be necessary. To diminish this time lag and to speed up the response time, so-called fast loops have been constructed (Fig. 2). A fast loop is a bypass in the process stream in which the fluid is propelled with increased velocity. By the introduction of a self-cleaning bypass filter, a virtually particulate-free sample stream can be introduced into the analyzer. The appropriate dimensions of the fast loop line depend on the delay time that can be accepted, on viscosity, etc., and can be calculated by means of the Hagen-Poisseuille equation.

In a preceding section, the influence of temperature was discussed. It would be possible to change the temperature by adjustment of the dimensions of the fast loop line in order to get the best possible heat exchange. However, there are two reasons to avoid such an approach: first, it will always be at
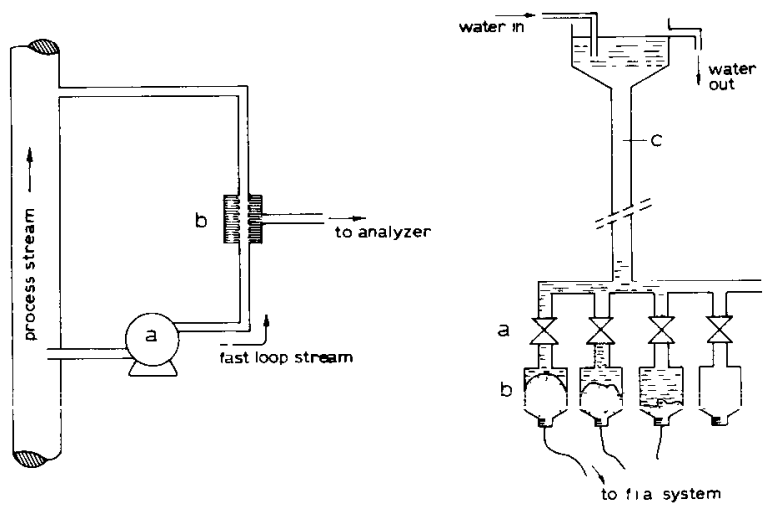

Fig. 2. Fast loop system: (a) pump; (b) self-cleaning bypass filter.

Fig. 3. Constant head device: (a) wide-bore water valve; (b) polythene bottle provided with flexible plastic bag containing carrier, reagent(s), ctc.; (c) wide-bore pipe 
the expense of transportation time, and secondly, from the process operation point of view, it might not be advisable to reintroduce a fast loop stream at a greatly different temperature into the main process stream. Therefore, any adjustment of temperature has to proceed in the sample stream between the fast loop system (e.g., bypass filter in Fig. 2) and the analyzer.

\section{COMPONENT PARTS OF A FLOW-INJECTION SYSTEM}

In a recent review on the application of flow-injection techniques to atomic absorption spectrometry (a.a.s.), Tyson [11] has made some noteworthy remarks on the components of flow-injection systems. Some of his remarks apply specifically to the use of a.a.s. as a detection system but others have a much broader scope, at least for applications in the laboratory. As discussed above, the situation may be somewhat different for process analyzers.

\section{Pumps}

It is common practice in f.i.a. to use multi-roller peristaltic pumps. The slight flow fluctuations that may occur are damped to some extent by the use of flexible and somewhat elastic tubing and, if necessary, an additional pulse-dampening device can be used. When in daily use, pump tubing gradually loses its flexibility, accompanied by a slow decrease in pumping capacity. Depending on the quality of the tubing, and the kind of fluids pumped through it, the tubes have to be replaced at certain intervals. Peristaltic pumps are sufficiently robust and reliable for use in process analysis. The vital parts can easily be protected against corrosive environmental attack. In an explosive environment, special precautions have to be taken to meet existing safety requirements.

The advantage of peristaltic and similar pumps is that they are capable, in principle, of maintaining a constant volumetric flow rate and correspondingly a constant residence time independent of minor changes in viscosity or variations in back-pressure because of restriction changes in the remainder of the system. This does not apply to the use of gas-pressurized reservoirs for reagents and carrier or to the use of constant-head vessels. However, both are cheap alternatives to pumping, and apart from the benefit of an almost completely pulse-free fluid flow, their main advantages are simplicity and the lack of any moving parts. As long as the geometry of the whole set-up is fixed and clogging or just narrowing of the conduits is avoided, a continuous and very constant flow can be guaranteed. In the author's laboratory, a flexible and essentially maintenance-free assembly has been tested (Fig. 3).

The same advantages apparently apply to the use of gas-pressurized reservoirs. They seem to be less attractive, however, because of the increased chance of formation of gas bubbles in the conduits; this is due to gradually decreasing gas solubility in proportion to the continuous pressure drop across the flow system. 


\section{Injection devices}

During the last decade, many home-made injection valves have been described. Many, particularly those from Bergamin's group [12], are ingenious but also rather complex. Most important from the point of view of process analysis is that such valves probably do not yet meet high standards of longterm reliability, and that valves which are not commercially available are of little interest for process analyzers.

At this moment a good, simple rotary valve with external sample loop seems to be a suitable choice. In tests, it has been proved that after 50000 switches, such valves still function properly, and no wear or tear was noticeable provided that the sample and carrier stream were virtually free from particles [13]. The "hydrodynamic" injection proposed by Růżicka and Hansen [14] is of interest for process analysis, but it has the drawback that two independent pumps are needed. Another valveless injection procedure, "controlled dispersion analysis" suggested by Sherwood et al. [15], seems less attractive for the purposes of process analysis.

\section{Detection systems}

It is beyond the scope of this article to review the types of sensing devices suitable for use in flow-injection systems. Only a few comments will be made on some general aspects of importance in process analysis. In addition to obvious criteria such as sensitivity, limit of detection and response time, the selection of an appropriate detection system strongly depends on factors that influence long-term uninterrupted operation. Media that exhibit a tendency to deposit formation will cause problems with optical detectors because deposits on optical windows will affect the transmission of light, and with electrochemical detectors because the nature or rate of electron-transfer reactions at the electrode surface can be changed. Thermistors are less sensitive to deposits and enthalpimetric detection should be of interest for this reason. The use of an enthalpimetric flow-through detector is being studied in the author's laboratory. Electrochemical as well as enthalpimetric detectors are essentially insensitive to turbidity or colour. For this reason, they should be preferable to spectrophotometric detectors for many applications.

The signals obtained from optical, enthalpimetric and some electrochemical (amperometric and conductometric) detectors exhibit a linear dependence on concentration. In these cases, the magnitude of the transient signal can be measured from the baseline, thus allowing for correction of drift. This does not apply to the important category of potentiometric detectors, which exhibit a logarithmic response. This means that it is not the peak height but the absolute value at the peak that is of interest. The baseline can be rather indeterminate and often shows large fluctuations. Because this can be rather confusing when low concentrations are involved, it is often recommended that the carrier stream be maintained at a relatively low but constant concentration of the species to be determined. Another possibility for circumventing this problem is computational on-line transformation of the potentials measured to their corresponding concentrations. 
To conclude this section, the concept and design of integrated microconduits recently introduced by Rủžixka and Hansen $[16,17]$ has to be mentioned. There is no doubt that this approach will contribute positively to the reliability of flow-injection systems; the possibility of replacement is a very attractive feature, comparable to the use of single boards or cards in microelectronics.

\section{PRACTICAL APPLICATIONS OF FLOW-INJECTION SYSTEMS IN PROCESS ANALYSIS}

Hardly any reference can be found to the industrial applications of f.i.a. for real continuous monitoring or control of processes. Even in a chapter entirely devoted to this subject, Ranger [9] does not give any example. Only very recently were some applications in the field of water quality surveillance reported. Gisin and Jardas [18] described a single-channel sequential monitoring method for phosphate and sulfate in industrial effluents by means of reversed f.i.a., using hydrodynamic injection and photometric detection. During a two-month run with a sampling frequency of 15 samples $\mathrm{h}^{-1}$, neither clogging nor significant baseline drift caused by deposition on the cell windows were observed because a proper wash cycle was used. Another unattended operation of f.i.a. was reported by Smith et al. [19]; by splitting the sample stream, nitrate, phosphate and sulfate in rivers could be monitored simultaneously. Finally, Petty and Johnson [20] described continuous monitoring by means of reversed f.i.a.; optimized methods were reported for the determination of nitrate, nitrite, phosphate, silicate, ammonia and primary amines.

In the author's laboratory, experience has been gathered with the longterm reliability of a flow-injection determination of sulfide in di-isopropanolamine (DIPA) solutions. Such DIPA or related amine solutions are used for the removal of hydrogen sulfide from, for instance, natural gas. The set-up, shown in Fig. 4, was used for several months in a test of suitable reactors. The use of the gas-diffusion membrane module provides selective transfer of some gaseous compounds from sample to detector stream but excludes contamination of the detector stream by all involatile compounds present in the sample stream. Detection was by a silver sulfide-based ion-selective electrode. The detector stream is forced through a nozzle at a short distance perpendicular to the electrode surface. By using such a wall-jet configuration a very short response time was obtained.

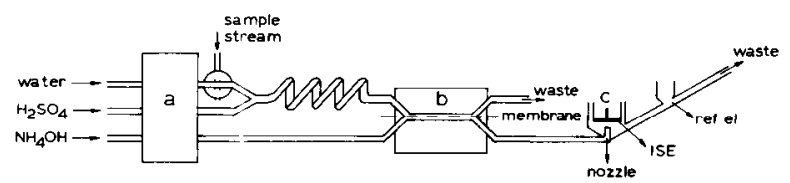

Fig 4. Assembly for the measurement of sulfide content of di-isopropylamine solution with potentiometric detection: (a) peristaltic pump; (b) membrane gas-diffusion module; (c) ion-selective sulfide electrode. 
The use of membranes for dialysis, gas diffusion, etc., deserves more attention particularly for on-line process analysis because it offers a very elegant way of avoiding interferences from many components and it protects the detector stream from undesirable contamination. As far as gas diffusion is concerned, it allows the determination of ammonia, carbon dioxide, cyanide (HCN) and sulfur dioxide (see e.g. [21]). Some preliminary experiments [22] have shown that hydrophilic microporous polypropylene membranes (Celgard 3501; Celanese Corp.) can be used in the determination of the water content of organic solvents. Water from the organic sample stream is transferred across the membrane to methanol and subsequently at the outlet of the membrane module is mixed with pyridine-free Karl Fischer reagent. Biamperometric detection in a flow-through cell is used.

\section{Conclusion}

Theoretically, f.i.a. has many features that should make it a valuable technique for on-line process control. The slow introduction in process analysis of flow injection-based analyzers, and indeed chemical analyzers in general, is caused by the stringent requirements for correct and uninterrupted operation. For some time to come, it is to be expected that applications will be found primarily in areas where possible failure of the monitoring function is unlikely to have serious short-term consequences. Surveillance of water quality can be considered as one such area.

\section{REFERENCES}

1 L. J. Wachel in D. P. Manka (Ed.), Automated Stream Analysis for Process Control, Academic Press, New York, 1984, Vol. 2, Chap. 15.

2 D. L. Massart, A. Dijkstra and L. Kaufman, Evaluation and Optımization of Laboratory Methods and Analytical Procedures, Elsevier, Amsterdam, 1978.

3 G. Kateman and F. W. Pijpers, Quality Control in Analytical Chemistry, Wiley, New York, 1981.

4 K. S. Johnson and R. L. Petty, Anal. Chem., 54 (1982) 1185.

5 A. G. Fogg and N. K. Bsebsu, Analyst (London), 109 (1984) 19.

6 A. Ríos, M. D. Luque de Castro and M. Valcárcel, Analyst (London), 109 (1984) 1487.

7 A. Fernández, M. A. Gómez-Nieto, M. D. Luque de Castro and M. Valcárcel, Anal. Chim. Acta, 165 (1984) 217.

8 E. L. Cussler, Diffusion; Mass Transfer in Fluid Systems, Cambridge University Press, Cambridge, 1984.

9 C. B. Ranger in D. P. Manka (Ed.), Automated Stream Analysis for Process Control, Academic Press, New York, 1982, Vol. 1, Chap. 2.

10 D. C. Cornish, G. Jepson and M. J. Smurthwaite, Sampling Systems for Process Analyzers, Butterworths, London, 1981, p. 3.

11 J. F. Tyson, Analyst (London), 110 (1985) 419.

12 E. A. G. Zagatto, A. O. Jacintho, L. C. R. Pessenda, F. J. Krug, B. F. Reis and H. Bergamin $F^{\circ}$., Anal. Chim. Acta, 125 (1981) 37.

$13 \mathrm{H}$. J. van den Dolder, private communication.

14 J. Ražižka and E. H. Hansen, Anal. Chim. Acta, 145 (1983) 1.

15 R. A. Sherwood, B. F. Rocks and C. Riley, Analyst (London), 110 (1985) 493.

16 J. Rǔžǐ̌ka and E. H. Hansen, Anal. Chim. Acta, 161 (1984) 1. 
17 J. Råżicka, Anal. Chem., 55 (1983) 1041A.

$18 \mathrm{M}$. Gisin and Z. Jardas, FACCS 11th Annual Meeting, Philadelphia, 1984, Abstr. no. 354 .

19 B. Smith, A. Sherry and A. Cherdak, F ACCS 11 th Annual Meeting, Philadelphia, 1984, Abstr. no. 355.

20 R. L. Petty and K. S. Johnson, FACCS 11th Annual Meeting, Philadelphia, 1984, Abstr. no. 356.

21 W. E. van der Linden, Anal. Chim. Acta, 151 (1983) 359.

22 L. M. M. Cristóva and W. E. van der Linden. Unpublished results. 